\title{
Successful Treatment of Refractory Immune Hemolysis Following Unrelated Cord Blood Transplant with Campath-1H
}

\author{
Mwe Mwe Chao, MD, ${ }^{1}$ John E. Levine, $M D,{ }^{1}$ James L. Ferrara, $M D,{ }^{1}$ Laura Cooling, $M D,{ }^{2}$ \\ Kenneth R. Cooke, $M D^{1}{ }^{1}$ Raymond J. Hutchinson, $\mathrm{MD}^{1}{ }^{1}$ and Gregory A. Yanik, $\mathrm{MD}^{1}{ }^{*}$
}

\begin{abstract}
Immune-mediated hemolytic anemia is a well-recognized complication of hematopoietic stem cell transplantation. We report on a 6-year-old boy with X-linked adrenoleukodystrophy who developed severe delayed alloimmune hemolytic anemia associated with immune-mediated neutropenia and thrombocytopenia following major $\mathrm{ABO}$ incompatible unrelated cord blood transplantation. The patient's cytopenias were refractory to treatment with corticosteroids, cyclosporine, intravenous immune globulin, rituximab, and
\end{abstract}

pentostatin. After one course of Campath-1H his hematologic parameters normalized, suggesting that the compound may be an effective therapy for complex immunohematologic disorders complicating hematopoietic stem cell transplantation. The case also emphasizes the importance of T-cells in transplant associated immune cytopenias. Pediatr Blood Cancer 2008;50:917-919.

(ㄷ) 2007 Wiley-Liss, Inc.

Key words: Campath-1H; cord blood transplantation; Evans syndrome; immune hemolytic anemia; X-linked adrenoleukodystrophy (ALD)

\section{INTRODUCTION}

Auto- or alloimmune-mediated hemolytic anemia occurs in $3-9 \%$ of allogeneic hematopoietic stem cell transplant (HSCT) patients $[1,2]$. In autoimmune hemolysis, donor-derived antibodies react against donor red blood cell ( $\mathrm{RBC}$ ) antigens, while in alloimmune hemolysis, RBC antigen incompatibility between donor and recipient gives rise to reactive alloantibodies [3]. The clinical course depends on whether the antibodies are auto or allo in origin and ranges from mild to life-threatening [3]. We describe a pediatric HSCT patient with refractory delayed alloimmune hemolytic anemia due to major $\mathrm{ABO}$ incompatibility that was further complicated by immune-mediated neutropenia and thrombocytopenia. He was successfully treated with Campath-1H following poor responses to multiple therapeutic agents.

\section{CASE REPORT}

A 6-year-old male with X-linked adrenoleukodystrophy (ALD) was treated with a $5 / 6$ mismatched, major $\mathrm{ABO}$ incompatible, unrelated cord blood (UCB) transplantation. The patient's ABO and HLA type were O-; A 24,68 B 40,44 DRB 0401,0701. The donor unit typed as group A+ with a class I disparity at A locus: A 24,32 B 40,44 DRB 0401,0701. In preparation for the transplant, he received busulfan, cyclophosphamide, and anti-thymocyte globulin. A total of $4.6 \times 10^{7}$ nucleated cells $/ \mathrm{kg}\left(1.8 \times 10^{5} \mathrm{CD} 34+\right.$ cells $\left./ \mathrm{kg}\right)$ were infused. Cyclosporine and methylprednisolone were given for graftversus-host disease (GVHD) prophylaxis. The patient engrafted neutrophils (absolute neutrophil count, ANC $>500 / \mathrm{mm}^{3}$ ) on day 20 . He became independent of packed red blood cells (PRBC) transfusions (hemoglobin $>8 \mathrm{~g} / \mathrm{dl}$ ) on day 56 and of platelet transfusions (platelets $>40,000 / \mathrm{mm}^{3}$ ) on day 70 . Peripheral blood PCR of microsatellite markers showed full donor engraftment on day 21.

Six months post-transplantation, the patient developed hemolytic anemia with a sudden decrease in hemoglobin to $7.1 \mathrm{~g} / \mathrm{dl}$ from $9.3 \mathrm{~g} / \mathrm{dl}$ and a corresponding increase in reticulocyte count $(6.42 \%)$, LDH (306 IU/L, normal ranges 140-280 IU/L), and indirect bilirubin $(1.4 \mathrm{mg} / \mathrm{dl})$ (Fig. 1). Direct antiglobulin testing (DAT) revealed $3+\operatorname{IgG}$ anti-A antibodies in the plasma and eluate, confirming the hemolysis was immune-mediated. Repeat engraftment analysis of peripheral blood demonstrated both donor (94\%) and recipient $(6 \%)$ cells.

The patient was started on methylprednisolone $(2 \mathrm{mg} / \mathrm{kg} / \mathrm{day})$ and the hemolysis initially improved. Two months later, while still receiving methylprednisolone $(0.5 \mathrm{mg} / \mathrm{kg} / \mathrm{day})$, the hemoglobin declined once again and the reticulocyte count, $\mathrm{LDH}$, and indirect bilirubin rose, prompting re-escalation of the methylprednisolone dose and initiation of rituximab therapy $\left(375 \mathrm{mg} / \mathrm{m}^{2}\right.$ IV weekly for four weeks $\times 2$ ). The patient's clinical course deteriorated with declining neutrophil and platelet counts over the next three months (Fig. 2). He required cytokine (erythropoietin, G-CSF, and GMCSF) support and 1-2 transfusions of PRBC (group O) and platelets (group A) per week. A bone marrow biopsy demonstrated normal cellularity with erythroid hypoplasia, granulocytic predominance, and adequate megakaryocytes. Evaluation for anti-neutrophil and anti-platelet antibodies was not undertaken. The patient then received pentostatin $(4 \mathrm{mg} / \mathrm{m} 2$ IV every two weeks $\times 2)$ without improvement. Nine months after the onset of hemolysis, Campath$1 \mathrm{H}$ (alemtuzumab, $15 \mathrm{mg}$ /day IV three times per week $\times 1$, Berlex Oncology, Montville, NJ) was administered. The patient tolerated Campath-1H therapy without adverse events. During the period of intensive immunosuppression the patient received pentamidine, fluconazole, acyclovir, and valganciclovir for infection prophylaxis.

Eight weeks after Campath-1H administration, the patient's hemoglobin increased to $8 \mathrm{~g} / \mathrm{dl}$ and platelet count increased to a value above $40,000 / \mathrm{mm}^{3}$ without support. His ANC rose above $1,000 / \mathrm{mm}^{3}$ without G-CSF or GM-CSF supplementation 18 weeks later. The patient's immunosuppressive medications were

\footnotetext{
${ }^{1}$ Department of Pediatrics, Division of Pediatric HematologyOncology and Blood and Marrow Transplantation, University of Michigan Health System, Ann Arbor, Michigan; ${ }^{2}$ Department of Laboratory Medicine and Pathology, University of Michigan Health System, Ann Arbor, Michigan

*Correspondence to: Gregory A. Yanik, University of Michigan Health System, 1500 East Medical Centre, 5303 CCGC, Ann Arbor, MI 48109-0914. E-mail: gyanik@med.umich.edu
}

Received 22 November 2006; Accepted 23 January 2007 


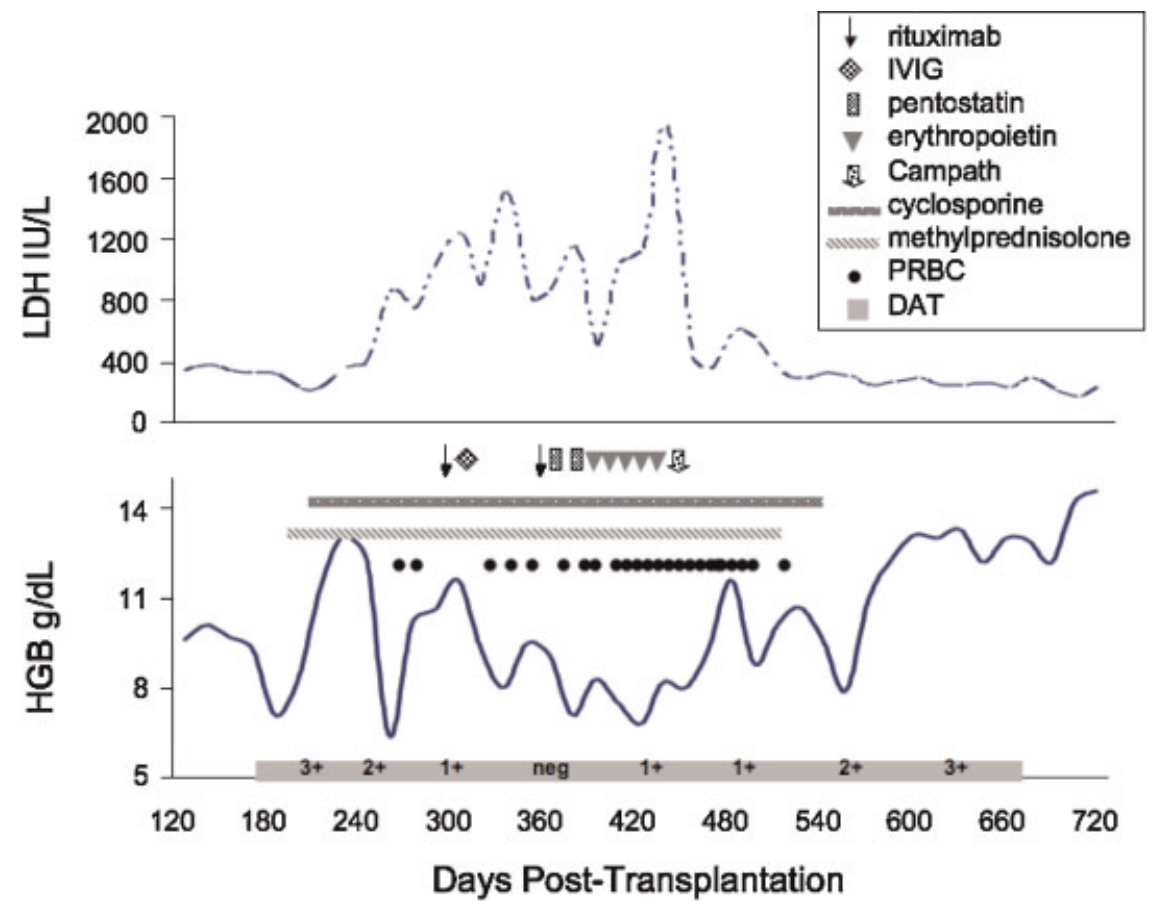

Fig. 1. Biochemical and hematologic evidence of hemolysis is demonstrated with a rising lactic dehydrogenase (LDH) level, falling hemoglobin $(\mathrm{Hgb})$ level, and positive direct antiglobulin testing (DAT). The patient was treated with several medications including rituximab, intravenous immunoglobulins (IVIG), pentostatin, cyclosporine, and methylprednisolone without response. Erythropoietin and packed red blood cell (PRBC) transfusions were also administered. After one course of Campath-1H therapy, the patient's hemoglobin improved and LDH normalized. Interestingly, the DAT remains positive despite a normal Hgb level.

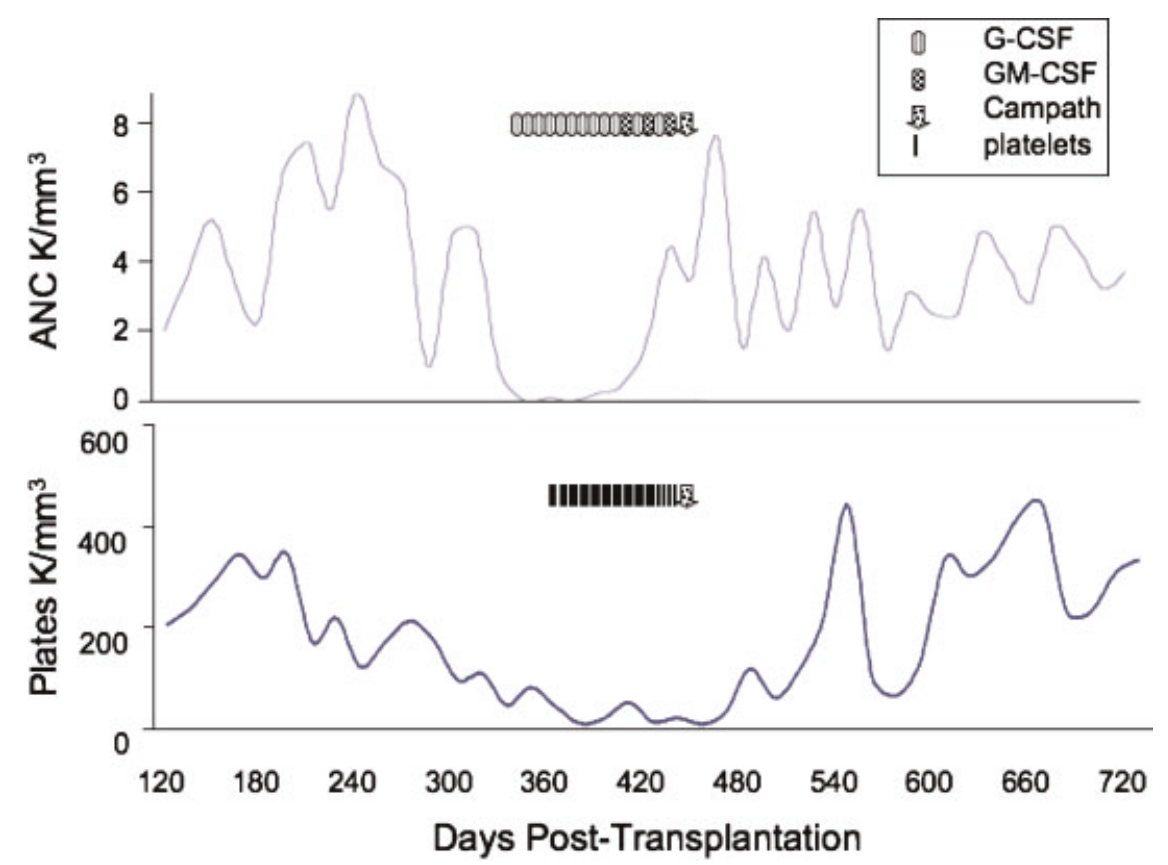

Fig. 2. The patient also developed immune-mediated thrombocytopenia and neutropenia. Platelets were transfused and granulocyte colony stimulating factor (G-CSF) and granulocyte-monocyte stimulating factor (GM-CSF) were administered for low absolute neutrophil count (ANC) levels. Both cytopenias resolved after Campath-1H therapy. Platelet and neutrophil values represented in the graph are pre-transfusion or precytokine values. 
discontinued with no signs of GVHD. He has maintained a hemoglobin $>12 \mathrm{~g} / \mathrm{dl}$, ANC $>2,000 / \mathrm{mm}^{3}$, and platelet count $>150,000 / \mathrm{mm}^{3}$ (Figs. 1 and 2) 22 months after Campath-1H treatment.

\section{DISCUSSION}

Major ABO incompatible HSCT is characterized by recipientderived $\mathrm{ABO}$ antibodies directed against donor $\mathrm{RBC}$ antigens. These anti-donor isohemagglutinins can cause immunohematologic complications including hemolysis, delayed erythrocyte engraftment, and pure RBC aplasia [4]. We present a patient who developed delayed alloimmune hemolytic anemia following mismatched major $\mathrm{ABO}$ incompatible UCB transplantation for X-linked ALD. The patient's pre-transplantation blood group was $\mathrm{O}-$ while the donor cord unit was A+. Detection of mixed chimerism and anti-A $\mathrm{RBC}$ antibodies suggested residual recipient lymphocytes produced anti-A antibodies responsible for hemolysis. Isohemagglutinins originating from enduring recipient lymphocytes have been previously reported [3]. We speculate our patient's relative immune competence in combination with a cyclosporine-based GVHD prophylaxis regimen predisposed to proliferation of recipient lymphocytes and generation of isohemagglutinins. O'Brien et al. showed that patients transplanted for metabolic disorders were 4.2 times more likely to experience autoimmune hemolysis in comparison with patients transplanted for malignancies. Patients with non-malignant disorders have not been exposed to cytotoxic or immunosuppressive therapy and are relatively immune competent which may contribute to immune hemolysis [2]. Moreover, although cyclosporine is immunosuppressive, it permits recipient lymphocyte proliferation and isohemagglutinin expansion; studies have shown that patients who receive cyclosporine GVHD prophylaxis are at increased risk of alloimmune hemolysis [1,3].

Our patient also developed neutropenia and thrombocytopenia. A bone marrow examination showed normal granulocyte and megakaryocyte numbers with maturation suggesting the low blood counts were immune-mediated. It is unclear whether anti-platelet and anti-neutrophil antibodies were present or were donor or recipient in origin. Based on studies in autoimmune hemolytic anemia associated with immune thrombocytopenia and/or neutropenia (Evans syndrome), the causative antibodies are directed against specific RBC, platelet, or neutrophil antigens and do not cross react. Decreased CD4 T-lymphocytes, increased CD8 Tlymphocytes, and a markedly decreased CD4:CD8 ratio in patients with Evans syndrome suggests that the disorder may be a result of abnormal T-cell regulation [5]. Furthermore, recent findings of poor clinical efficacy of rituximab for the treatment of autoimmune neutropenia and pure red cell aplasia in comparison with historical responses to Campath-1H implies a critical role for T-cells in controlling the expansion of autoreactive B-cells [6]. Response to Campath-1H therapy supports a contributory if not causal role for T-cell dysregulation in our patient's cytopenias.

Campath- $1 \mathrm{H}$ is a monoclonal antibody specific for CD52 surface glycoprotein expressed on B- and T-lymphocytes [7]. Binding of
Campath-1H to CD52 antigen activates antibody-dependent cellmediated lysis of cells and profound lymphopenia [8]. While Blymphocytes recover by six months [7], full recovery of CD4 and CD8 T-lymphocytes can take more than 12 months [8]. Because of these properties, Campath- $1 \mathrm{H}$ has been used for treatment of immune-mediated disorders including autoimmune cytopenias. Willis et al. administered Campath- $1 \mathrm{H}$ to 21 patients with refractory autoimmune hemolytic anemia, pure red cell aplasia, immune thrombocytopenia purpura, autoimmine hemolytic anemia with thrombocytopenia, or autoimmune pancytopenia and found a $71 \%$ initial response rate and a $29 \%$ sustained response rate; concluding that Campath- $1 \mathrm{H}$ is an alternative therapeutic option for resistant autoimmune cytopenias [7].

Complex immunohematological events can complicate HSCT. Imbalances in reactive and regulatory donor and recipient lymphocytes may underlie our patient's cytopenias. Successful treatment of immune-mediated hemolytic anemia, neutropenia, and thrombocytopenia with Campath- $1 \mathrm{H}$ in our patient suggests that this antibody may terminate pathologic immune processes. As T-lymphocytes are major targets of Campath-1H, our case also highlights an important role for T-lymphocytes in the pathophysiology of immune-mediated cytopenias.

\section{ACKNOWLEDGMENT}

Special thanks to Dr. Christian Kratz for his helpful suggestions and review of the manuscript.

\section{REFERENCES}

1. Sniecinski IJ, Oien L, Petz LD, et al. Immunohematologic consequences of major abo-mismatched bone marrow transplantation. Transplantation 1988;45:530-534.

2. O'Brien TA, Eastlund T, Peters C, et al. Autoimmune haemolytic anaemia complicating haematopoietic cell transplantation in paediatric patients: High incidence and significant mortality in unrelated donor transplants for non-malignant diseases. $\mathrm{Br}$ J Haematol 2004;127:67-75.

3. Petz LD. Immune hemolysis associated with transplantation. Semin Hematol 2005;42:145-155.

4. O'Donnell MR. Blood group incompatibilities and hemolytic complications of hematopoietic cell transplantation. In: Blume KG, Forman SJ, Stephen J, et al. editors. Thomas' hematopoietic cell transplantation. Oxford: Blackwell Publishing; 2004. pp 824831.

5. Norton A, Roberts I, Management of Evans syndrome. $\mathrm{Br}$ J Haematol 2006;132:125-137.

6. Dungarwalla M, Marsh JC, Tooze JA, et al. Lack of clinical efficacy of rituximab in the treatment of autoimmune neutropenia and pure red cell aplasia: Implications for their pathophysiology. Ann Hematol 2006;86:191-197.

7. Willis F, Marsh JC, Bevan DH, et al. The effect of treatment with campath- $1 \mathrm{~h}$ in patients with autoimmune cytopenias. $\mathrm{Br}$ J Haematol 2001;114:891-898.

8. Berlex Oncology. Campath (alemtuzumab) [package insert]. Montville, NJ: 2005. 